\title{
QUASI-FUCHSIAN SURFACES IN HYPERBOLIC KNOT COMPLEMENTS
}

\author{
COLIN C. ADAMS and ALAN W. REID
}

(Received 8 August 1992)

Communicated by J. H. Rubinstein

\begin{abstract}
Examples of hyperbolic knots in $S^{3}$ are given such that their complements contain quasi-Fuchsian non-Fuchsian surfaces. In particular, this proves that there are hyperbolic knots that are not toroidally alternating.
\end{abstract}

1991 Mathematics subject classification (Amer. Math. Soc.): 57 N 10, 57 M 50.

\section{Introduction}

In the following, an essential surface in a compact orientable 3-manifold $M$ shall mean a properly embedded incompressible, boundary incompressible, compact orientable surface in $M$ that is not boundary parallel.

Given a compact orientable 3-manifold $M$ with non-empty boundary and with hyperbolic interior, and a closed essential surface embedded within it, it is of interest to determine the geometry inherited by the surface. Such surfaces are either quasi-Fuchsian, meaning that, the limit set for the representation of the surface group into $\operatorname{PSL}(2, \mathrm{C})$ induced by the hyperbolic structure on $M$ is a topological circle, or they contain an accidental parabolic element, meaning that, there is a loop on the surface that lifts to a parabolic isometry of $H^{3}$ under the induced representation of the surface group. We remark that it follows from the

(C) 1993 Australian Mathematical Society 0263-6115/93\$A2.00+0.00

The first author was supported by NSF Grant DMS-9000937. The second author was supported by NSF Grants DMS-8505550, administered by MSRI, and DMS-9108050. 
classical theory of Kleinian groups that the existence of an accidental parabolic element in a surface group implies a simple accidental parabolic loop. Indeed, the only possibilities other than simple loops are powers of the appropriate primitive element in the fundamental group of the surface, $\mathrm{cf}$. [10, Chapter 9D].

A particularly simple class of hyperbolic structure within the class of quasiFuchsian structures is that of a totally geodesic surface. In this case the surface group is a Fuchsian group and hence stabilizes a geometric circle.

This paper is concerned with the case of a closed essential surface in a hyperbolic knot exterior in $S^{3}$. Large classes of hyperbolic knots and links in $S^{3}$ are known to contain closed essential surfaces in their complements. See for example $[8,9,15]$. However, all of these constructions yield surfaces that contain accidental parabolic elements. In [11], it was shown that every closed incompressible surface in the complement of an alternating hyperbolic link complement in the 3-sphere contains an accidental parabolic curve. This was extended to hyperbolic almost alternating knots in [2] and hyperbolic toroidally alternating knots in [1]. These examples include all but at most three of the hyperbolic knots of eleven or fewer crossings appearing in [4].

It was conjectured in [11] that a hyperbolic knot in $S^{3}$ cannot contain a closed embedded totally geodesic surface in its complement. In fact, up to this point, an explicit example of even a quasi-Fuchsian surface in a hyperbolic knot complement has not been given. One of the aims of this paper is to give a fairly general construction for such examples. We will then prove that for most of the examples given, the surfaces generated are quasi-Fuchsian surfaces that are not Fuchsian, and hence do not contradict the conjecture of [11].

We remark that examples of link complements in $S^{3}$ that contain a closed essential totally geodesic surface are known, cf. [12], and there are examples of hyperbolic knot exteriors in closed 3-manifolds other than $S^{3}$ that do contain a closed essential totally geodesic surface, cf. [13].

These examples of knot complements containing quasi-Fuchsian surfaces also provide the first examples of hyperbolic knots in $S^{3}$ that are known to fall outside the category of toroidally alternating knots. A toroidally alternating knot is defined to be a knot that can be projected onto a standardly embedded torus in $S^{3}$ such that the projection is alternating when viewed from one side of the torus and such that every non-trivial curve on the torus intersects the projection. In [1], it was proved that a toroidally alternating knot in $S^{3}$ contains no incompressible meridianally incompressible surfaces in its complement. However, an incompressible surface with no accidental parabolic elements must be meridianally incompressible. Hence a knot that contains an incompressible surface 
with no accidental parabolic elements in its complement cannot be toroidally alternating.

Extensive use has been made of SNAPPEA, the computer program developed by Jeff Weeks that computes hyperbolic structures on 3-manifolds. See [18] for more information on this remarkable program. Thanks to Allen Hatcher and Jeff Weeks for helpful conversations.

\section{Knots in handlebodies}

The basic fact that underlies our construction is the following.

OBSERVATION 2.1. Let $K$ be a knot in a genus $g$ handlebody $H$, where $g \geq 2$. Let $W$ be the complement of the interior of a genus $g$ handlebody in $S^{3}$. If $K$ has been chosen so that $H-K$ is hyperbolic and $H-N(K)$ contains no essential annuli running from the surface to the knot, and if $W$ has been chosen to be hyperbolic and contain no essential annuli, then we can choose a way to glue $\partial H$ to $\partial W$ in order to obtain a knot complement in $S^{3}$ that is hyperbolic and that contains a genus $g$ closed essential quasi-Fuchsian surface.

PROOF. This is almost a tautology following from classical theory. An essential annulus running from the knot to the surface corresponds to an accidental parabolic loop. As noted above, the existence of an accidental parabolic loop implies the existence of a simple accidental parabolic loop and therefore, by the annulus theorem, there can be no immersed annulus running from the knot to the surface. Hence the surface is quasi-Fuchsian in $H-N(K)$.

The assumption on $W$ implies there is no annulus running from the surface back to itself. Therefore the surface is acylindrical in $W$. On gluing $H-N(K)$ to $W$ as prescribed, we get a hyperbolic knot complement with a closed essential quasi-Fuchsian surface.

If we further insist that in $H-N(K)$ the surface is also acylindrical, that is, there is no annulus running from the surface back to itself, then the resultant closed surface will be acylindrical.

We now proceed to construct explicit examples, with a view to making them as simple as possible. In this regard, it would be interesting to compute the smallest volume of a hyperbolic knot complement containing a closed essential quasi-Fuchsian surface. 


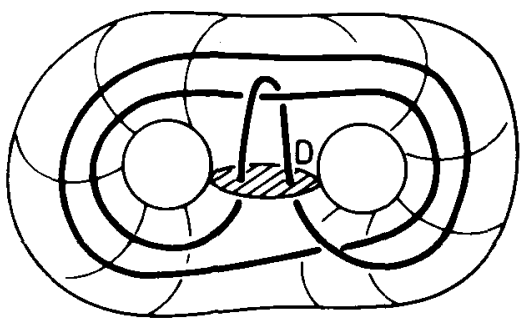

(a)

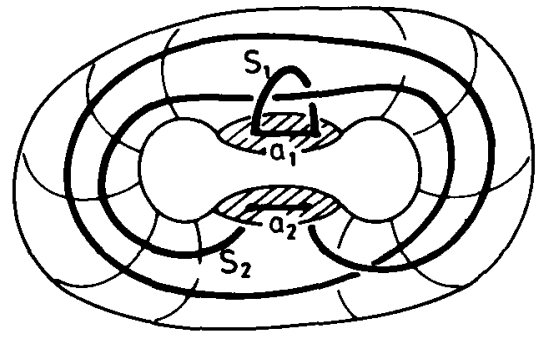

(b)

FIGURE 1

We begin with the knot $K_{1}$ in the genus two handlebody $H$ that appears in Figure 1. This example was suggested by Allen Hatcher.

LEMMA 2.2. Int $\left(H-N\left(K_{1}\right)\right)$ is hyperbolic and $\partial\left(H-N\left(K_{1}\right)\right)$ contains no accidental parabolic elements. However, $H-N\left(K_{1}\right)$ does contain an essential annulus with both boundary components in $\partial H$.

PROOF. In order to show that $H-K_{1}$ is hyperbolic, the work of Thurston (cf. [16]) implies that it is enough to show that $H-N\left(K_{1}\right)$ is irreducible, has incompressible boundary, and contains no essential torus. In order to prove $\partial H$ contains no accidental parabolic elements, it is enough to show that there is no essential annulus with at least one boundary component in $\partial N\left(K_{1}\right)$.

That $H-N\left(K_{1}\right)$ is irreducible is immediate, as the knot represents a nontrivial element in $\pi_{1}(H)$ and hence could not be contained in a 3-ball.

Let $D$ be the twice-punctured disk depicted in Figure 1a. For convenience, we will also denote by $D$ the disk with two holes obtained from this disk when the interior of a neighborhood of $K_{1}$ is removed. Let $H^{\prime}$ be the solid torus that results when we cut $H$ open along $D$. Let $D_{1}$ and $D_{2}$ be the two copies of $D$ on the boundary of $H^{\prime}$. We will use $F$ to denote the surface in either $H$ or $H^{\prime}$. Let $s_{1}, s_{2}, a_{1}$ and $a_{2}$ be the four arcs depicted in Figure $1 \mathrm{~b}$, and let $q_{1}=s_{1} \cup a_{1}, q_{2}=s_{2} \cup a_{2}$. Then $q_{1}$ and $q_{2}$ are non-trivially linked and $q_{2}$ wraps twice longitudinally around $H^{\prime}$. Notice that $H-N\left(K_{1}\right)$ contains no essential torus that does not intersect $D$, for such a torus would be essential in $H^{\prime}-N\left(s_{1} \cup s_{2}\right)$, which is impossible.

Let $F$ be a properly embedded disk, annulus or torus in $H-N\left(K_{1}\right)$ that is essential. Since $H^{\prime}-N\left(K_{1}\right)$ is a handlebody, and since $F \cap H^{\prime}$ is incompressible in $H^{\prime}-N(K)$, it follows that $F \cap H^{\prime}$ must be a set of disks and annuli. 
Let $(d, e)$ measure the complexity of the intersection of $F$ and $D$, where $d$ is the number of intersections of $\partial F$ with $\partial D$ (when we remove int $\left(N\left(K_{1}\right)\right.$ ) from $D$ ) and $e$ is the number of simple closed curves and arcs in $F \cap D$. Isotope $F$ to minimize $(d, e)$ lexicographically.

Suppose first that there is a disk component $D^{\prime}$ in $F \cap H^{\prime}$ with boundary a simple closed curve in $\operatorname{int}(F)$. Then its boundary is contained in $D$. Hence it must cut a 3-ball from $H^{\prime}$. If the 3-ball contains $s_{1}$ or $s_{2}$, it will contain $q_{1}$ or $q_{2}$ respectively. As $q_{1}$ and $q_{2}$ are linked, it must contain both or neither of them. It cannot contain both, as the boundary of $D^{\prime}$ is contained in either $D_{1}$ or $D_{2}$. Hence, $D^{\prime}$ can be isotoped through $D$, contradicting our assumption that $(d, e)$ is minimal.

Suppose now that $D^{\prime}$ is a disk component of $F \cap H^{\prime}$ such that one connected arc of its boundary lies in $D$ and the remaining arc of its boundary lies in $\partial H$. Since $H^{\prime}$ is a solid torus, $D^{\prime}$ is either a meridianal disk in $H^{\prime}$ or it cuts a 3-ball from $H^{\prime}$. In the first case, the algebraic intersection number of $q_{2}$ with $D^{\prime}$ must be two. Since $s_{2}$ cannot intersect $D^{\prime}, a_{2}$ must intersect $\partial D^{\prime}$ twice algebraically. However, the boundary of $D^{\prime}$ only intersects $D$ in a single connected arc, making this impossible.

If, on the other hand, $D^{\prime}$ cuts a 3-ball $B$ from $H^{\prime}$, the boundary of $D^{\prime}$ is a trivial curve on $\partial H^{\prime}$ bounding a disk $D^{\prime \prime}$. The desk $D^{\prime \prime}$ cannot contain just one of the two endpoints of either of the strings $s_{1}$ and $s_{2}$. The $\operatorname{arc}$ of $\partial D^{\prime}-\left(\operatorname{int}\left(D_{1} \cup D_{2}\right)\right)$ either cuts a disk from $\partial H^{\prime}-\left(\operatorname{int}\left(D_{1} \cup D_{2}\right)\right)$ or it does not. In the first case, we can isotope $D^{\prime}$ to lower the number $d$, a contradiction. In the second case, $D^{\prime \prime}$ contains all of one of the two disks $D_{1}$ or $D_{2}$. Hence the 3-ball $B$ must contain all of either $q_{1}$ or $q_{2}$. Since these two knots in $H^{\prime}$ are linked, $B$ must contain both of them. However, $q_{2}$ wraps twice longitudinally around $H^{\prime}$ and therefore cannot be contained in a 3-ball within $H^{\prime}$.

Suppose now that $D^{\prime}$ is a disk component of $F \cap H^{\prime}$ such that one connected arc of its boundary lies in $D$ and the remaining arc of its boundary lies in $\partial N\left(K_{1}\right)$. The arc that lies in $\partial N\left(K_{1}\right)$ must be parallel through $N\left(K_{1}\right)$ to either $s_{1}$ or $s_{2}$, as otherwise we could lower $d$. However, $D^{\prime}$ then provides us with either an isotopy of string $s_{1}$ to an $\operatorname{arc}$ in $D_{1}$ or an isotopy of string $s_{2}$ to an arc in $D_{2}$. As the only arc from one endpoint of $s_{i}$ to the other in $D_{i}$ is $a_{i}$, up to isotopy, the existence of $D^{\prime}$ implies that $q_{i}$ is trivial in $H^{\prime}-q_{j}$ for $j \neq i$. However, $q_{1}$ links $q_{2}$, a contradiction.

In the case that $F$ is a disk, the only possibility remaining is that $F$ does not intersect $D$. Since $F$ will not intersect the knot $q_{2}$ that wraps twice longitudinally around $H^{\prime}$, it must be that the boundary of $F$ is trivial on $\partial H^{\prime}$. The only way that 
$F$ could be a compressing disk for $\partial H$ is if $\partial F$ cuts a disk off $\partial H^{\prime}$ that contains one or both of $D_{1}$ and $D_{2}$. In this case, $F$ cuts a 3-ball off $H^{\prime}$ that contains one or both of $D_{1}$ and $D_{2}$. In this case, $F$ cuts a 3-ball off $H^{\prime}$ that contains at least one of $q_{1}$ or $q_{2}$. As they are linked, it must contain both, but as we have already mentioned, a 3-ball in $H^{\prime}$ cannot contain $q_{2}$. This concludes the proof that $\partial H$ is incompressible in $H-N\left(K_{1}\right)$.

We next deal with annuli in $F \cap H^{\prime}$. Suppose first that there is an annulus component $A$ of $F \cap H^{\prime}$ with a boundary component that is meridianal on $\partial N\left(K_{1}\right)$. If $A$ has two meridianal boundary components on $\partial N\left(K_{1}\right)$, then $A$ can be extended to a twice-punctured sphere in $H^{\prime}-\left(s_{1} \cup s_{2}\right)$. As each string is unknotted, $A$ is then parallel to an annulus in $\partial N\left(K_{1}\right)$. If only one boundary component of $A$ is a meridian on $\partial N\left(K_{1}\right)$, then $A$ can be extended to a once-punctured disk $E$ properly embedded in $H^{\prime}-\left(s_{1} \cup s_{2}\right)$. By the previous elimination of possibilities for disks in $F \cap H^{\prime}$, the boundary of $E$ either lies entirely in $D_{1}$ or $D_{2}$ or it lies in $\partial H^{\prime}-\left(D_{1} \cup D_{2}\right)$. Then $E$ cannot be a meridianal disk in $H^{\prime}$ as it intersects $q_{2}$ at most once. Hence, $E$ cuts a 3-ball from $H^{\prime}$. In order that the number of strings entering the 3-ball is even, it must be that the boundary of $E$ bounds a disk $G$ in $\partial H^{\prime}$ containing an odd number of endpoints of strings. Hence, $\partial E$ must be contained entirely within one of the disks $D_{i}$ and the other disk $G$ must contain exactly one endpoint of $s_{1} \cup s_{2}$. However, $E \cup G$ then forms a sphere containing an unknotted strand of an $s_{i}$. Hence, $A$ can be isotoped to lower $d$.

Suppose now that a component $A$ of $F \cap H^{\prime}$ is an annulus that does not have a boundary component that is meridianal on $\partial N\left(K_{1}\right)$. By the cases that we have already eliminated, it must be that each boundary component of the annulus either lies entirely in $D_{1}$ or $D_{2}$ or it lies entirely in $\partial H^{\prime}-\left(D_{1} \cup D_{2}\right)$. Suppose first that both boundary components of $A$ are trivial on $\partial H^{\prime}$ and the two disks that these trivial boundary components bound on $\partial H^{\prime}$ are disjoint. Then by irreducibility of $H^{\prime}, A$ cuts a 3-ball from $H^{\prime}$. For each $i$, it must either contain both endpoints of $s_{i}$ in its boundary or neither endpoint. Suppose that it contains both endpoints of $s_{i}$ in its boundary such that they both lie in the disk bounded by one boundary component of $A$. Then $q_{i}$ is contained in the 3-ball. Since $q_{i}$ is either completely contained in the 3-ball or does not intersect the 3-ball, and since $q_{i}$ and $q_{j}$ are linked, $q_{j}$ is also contained in the 3-ball. But $q_{2}$ cannot be in any such 3-ball, a contradiction.

Hence, if either endpoint of $s_{i}$ is contained in the boundary of the 3-ball, the two endpoints must be contained in disks bounded by the two distinct boundary components of $A$. However, this implies that both boundary components of $A$ 
are contained in a particular $D_{i}$. Hence $s_{j}$ is not contained in $B$, for $j \neq i$. However, we then have the unknotted arc $s_{i}$ contained in the 3-ball $B$, so it must be that the annulus $A$ is parallel to the string $s_{i}$. Such annuli can only glue together to form a boundary-parallel torus, and as we will eliminate all other possibilities for $F \cap H^{\prime}$, an essential surface is not generated.

Suppose now that the boundary components of $A$ are still trivial in $\partial H^{\prime}$ but the disks that they bound on $\partial H^{\prime}$ are concentric, one containing the other. The irreducibility of $H^{\prime}$ implies $A$ either cuts a non-trivial knot exterior from $H^{\prime}$ or $A$ cuts $H^{\prime}$ into two solid tori $V_{1}$ and $V_{2}$. In the first case, the incompressibility of $A$ would imply that the boundary of the knot exterior was incompressible in $H^{\prime}-N\left(K_{1}\right)$. However, the fundamental group of $H^{\prime}-N\left(K_{1}\right)$ is free and therefore cannot contain a subgroup isomorphic to the fundamental group of a non-trivial knot exterior.

In the second case, let $V_{2}$ be the solid torus such that its boundary contains the disk bounded by the innermost boundary component of $A$. The incompressibility of $A$ implies that at least one endpoint of an $s_{i}$ lies within this inner disk. If the other endpoint does not lie within the inner disk, it must lie outside the larger disk. However, then both boundary components of $A$ lie within $D_{i}$ and $A$ can be isotoped through $D_{i}$ to lower $e$.

Therefore there must be an $s_{i}$ such that both of its endpoints are contained in the inner disk. The endpoints of $s_{j}$ for $j \neq i$ must both either lie in the inner disk, outside the outer disk or in the outer disk but not in the inner disk. In the first two cases, we can either isotope $F$ to lower $(d, e)$, or $F$ is boundary-compressible in $\partial H$. In the last case, each of $V_{1}$ and $V_{2}$ contain one of $q_{1}$ or $q_{2}$. Since $q_{2}$ wraps twice longitudinally around $H^{\prime}$, and since $V_{1}$ is contained in a 3-ball in $H^{\prime}$, it must be that $q_{2}$ is contained in $V_{2}$ and $q_{1}$ is contained in $V_{1}$. However, we could then isotope $A$ through $H^{\prime}-q_{2}$ to $\partial H^{\prime}$ by pushing it through $V_{1}$, isotoping $q_{1}$ to $\partial H^{\prime}$ in the process. This contradicts the fact $q_{1}$ and $q_{2}$ are linked once while $q_{2}$ wraps twice longitudinally around $H^{\prime}$.

Suppose now that one boundary component of $A$ is trivial on $\partial H^{\prime}$ and the other is not. Then the non-trivial boundary component must be meridianal on $\partial H^{\prime}$, as it is isotopic to a trivial component. Let $D^{\prime \prime}$ be the disk on $\partial H^{\prime}$ bounded by the trivial boundary component of $A$. Then $A \cup D^{\prime \prime}$ is a meridianal disk of $H^{\prime}$. Hence $q_{2}$ must intersect it twice algebraically. However, $q_{2}$ will not intersect $A$ and if it intersects $D^{\prime \prime}$, it can be pushed off slightly to intersect it at most once, a contradiction.

The last possibility for $A$ is that both boundary components are non-trivial on $\partial H^{\prime}$. Then the boundary components must be contained in $\partial H^{\prime}-\left(D_{1} \cup D_{2}\right)$, 


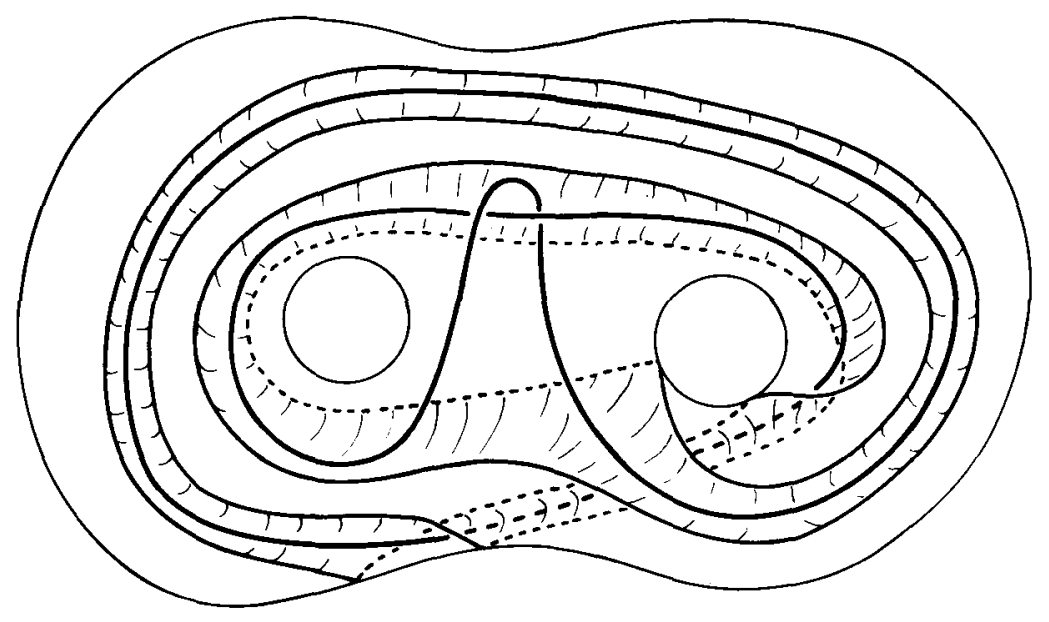

FIGURE 2

implying that $F$ is the annulus $A$, with both boundary components in $\partial H$. This case does occur, and provides an essential annulus in $H-N\left(K_{1}\right)$ when both boundary components are $(1,2)$-curves on $\partial H^{\prime}$. We draw such an annulus in Figure 2.

The only remaining possibility for the essential surface $F$ is if it is an annulus such that it intersects $H^{\prime}$ in disks, each disk of which has four arcs on its boundary, two of which are in $D_{1} \cup D_{2}$.

It cannot be the case that there exists an essential annulus with both boundary components in $\partial N\left(K_{1}\right)$, as the existence of such an annulus would imply the existence of an essential torus, a case we have already eliminated.

Suppose that $F$ is an essential annulus with one boundary component in $\partial H$ and the other in $\partial N\left(K_{1}\right)$. Each disk in $F \cap H^{\prime}$ has two connected arcs in $D_{1} \cup D_{2}$, one arc in $\partial N\left(K_{1}\right)$ and one arc in $\partial H^{\prime}-\left(D_{1} \cup D_{2}\right)$. The arc in $\partial N\left(K_{1}\right)$ is parallel through $N\left(K_{1}\right)$ to either $s_{1}$ or $s_{2}$. There is at least one such disk for each of $s_{1}$ and $s_{2}$. Let $D^{\prime}$ be such a disk for $s_{1}$. Then, $D^{\prime}$ provides an isotopy of $s_{1}$ to an $\operatorname{arc} a_{1}^{\prime}$ in $\partial H^{\prime}$. Since $s_{1}$ is already isotopic in $a_{1}$, we have that $a_{1}$ is homotopic to $a_{1}^{\prime}$, so $a_{1} \cup a_{1}^{\prime}$ is homotopically trivial in $H^{\prime}-q_{2}$. Since $a_{1} \cup a_{1}^{\prime}$ crosses $\partial D_{1}$ at only two points, it cannot be a homotopically meridianal curve on $\partial H^{\prime}$. Since $q_{2}$ wraps twice longitudinally around $H^{\prime}, a_{1}^{\prime}-D_{1}$ must cut a disk from $\partial H^{\prime}-\left(D_{1} \cup D_{2}\right)$. However, we can then isotope $F$ to lower $d$, a contradiction. 


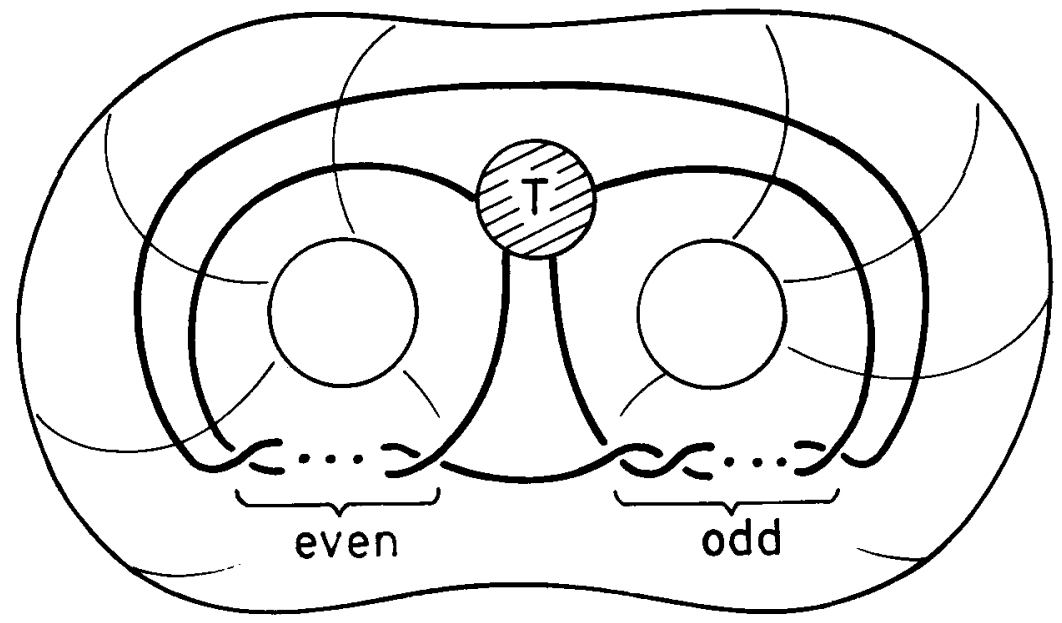

FIGURE 3

Note that the above proof could be extended to apply to any knot of the form in Figure 3, where $T$ is a tangle such that the bottom two strands in the tangle are connected to one another in the tangle, and similarly for the top two strands, and $T$ makes the two knots $q_{1}$ and $q_{2}$ (as in Figure $1 \mathrm{~b}$ ) into a non-splittable link.

Our next example of a knot complement in a handlebody does not contain any essential annulus.

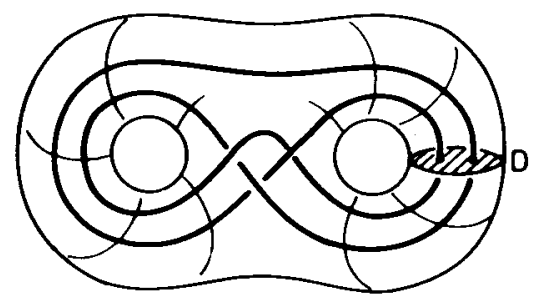

(a)

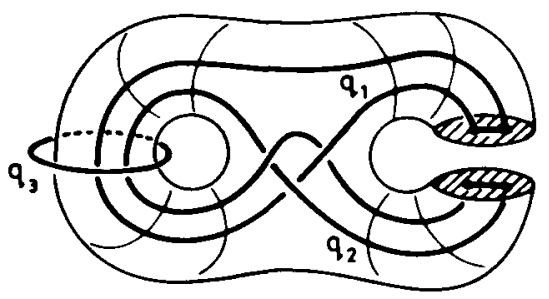

(b)

FIGURE 4

LEMMA 2.3. If $\mathrm{K}_{2}$ is the knot appearing in the handlebody $\mathrm{H}$ in Figure 4, then $\mathrm{H}-\mathrm{N}\left(\mathrm{K}_{2}\right)$ has hyperbolic interior and contains no essential annulus.

ProOF. Let $F$ be an essential disk, annulus or torus in $\mathrm{H}-\mathrm{N}\left(\mathrm{K}_{2}\right)$. Utilizing the disk $D$ in Figure 4, together with the knots $q_{1}, q_{2}$ and $q_{3}$, we can mimic 
the arguments in the proof of Lemma 2.1 to prove hyperbolicity. We can also eliminate all of the annular components of $F \cap H^{\prime}$ with the possible exception of an annulus such that both its boundary components are non-trivial curves on $\partial H^{\prime}$. These non-trivial curves must lie in $\partial H^{\prime}-\left(D_{1} \cup D_{2}\right)$.

Suppose first that the two boundary components of $A$ are meridianal on $\partial H^{\prime}$. They cut the boundary of $H^{\prime}$ into two annuli. If either annulus does not contain one of $D_{1}$ or $D_{2}$, then either $A$ is boundary-parallel into the boundary of $H^{\prime}-\left(D_{1} \cup D_{2}\right)$ or we can construct an incompressible torus in $H-N\left(K_{2}\right)$, contradicting our elimination of these tori. Hence, $A$ must separate $q_{1}$ from $q_{2}$. However, as both $q_{1}$ and $q_{2}$ are individually longitudinal in $H^{\prime}$, no annulus with meridianal boundary components can separate them.

Suppose now that the two boundary components of $A$ are not meridianal on $\partial H^{\prime}$. An annulus of this type cuts a solid torus from $H^{\prime}$ that must contain one of $q_{1}$ or $q_{2}$ so that it is not boundary compressible. However, if it does not contain both of $q_{1}$ and $q_{2}$, we could use it to homotope one to the boundary of $H^{\prime}$ in the complement of the other, a contradiction. Hence, it must contain both $q_{1}$ and $q_{2}$. Since we can then use it to homotope both of them into a $(p, q)$-annulus on the boundary of $H^{\prime}$, it must be that $q= \pm 1$. However, the annulus is then boundary-compressible to the side that does not contain $q_{1}$ and $q_{2}$.

As we did in the proof of Lemma 2.2, we can also eliminate all disk components $D^{\prime}$ of $F \cap H^{\prime}$ except for disks such that their boundaries contain two arcs in $D$ and either one arc in $\partial N\left(K_{2}\right)$ and the other arc in $\partial H^{\prime}-\left(D_{1} \cup D_{2}\right)$ or two arcs in $\partial H^{\prime}-\left(D_{1} \cup D_{2}\right)$. In both these cases, $F$ is an essential annulus.

In the first case, as we saw in the proof of Lemma $2.2, F$ is an annulus and there is at least one such disk for each of $s_{1}$ and $s_{2}$, yielding an isotopy of $s_{i}$ to $\partial H^{\prime}$ in the complement of $q_{j}, j \neq i$. However, $q_{j}$ links $s_{i}$, and so such an isotopy is not possible.

In the second case, $\partial D^{\prime}$ cannot be meridianal on $H^{\prime}$ as if it were, since $D^{\prime}$ must avoid $s_{1}$ and $s_{2}$ the boundary of $D^{\prime}$ would be forced to intersect $D_{1}$ and $D_{2}$ more than once each. Otherwise, $D^{\prime}$ must cut a 3-ball off $H^{\prime}$. Each arc of $\partial D^{\prime} \cap\left(D_{1} \cup D_{2}\right)$ must be an essential arc in $D_{1} \cup D_{2}$ so that the annulus $F$ is not boundary compressible. However, since $\partial D^{\prime}$ cannot separate the endpoints of an $s_{i}$, both arcs must lie in the same $D_{i}$. Since $D^{\prime}$ does not intersect $q_{j}$, and since $q_{j}$ is longitudinal, $q_{j}$ must lie to the side of $D^{\prime}$ that is not a 3-ball. If $s_{i}$ lies to that side also, then we could lower $(d, e)$ by isotoping $D^{\prime}$ through $D$. Thus, $s_{i}$ must be an unknotted arc in the 3-ball. However, we could then isotope $s_{i}$ to $\partial H_{i}$ through the 3-ball, contradicting the fact that $q_{j}$ links $s_{i}$. 


\section{Knotted handlebodies}

Now, we would like to embed either of our two possibilities for $(H, K)$ in the 3-sphere, so that the complement of $\operatorname{int}(H)$ is itself a hyperbolic 3-manifold with no essential annuli. There are several candidates for $S^{3}-H$. In particular, the two handlebody complements obtained by removing the interiors of regular neighborhoods of the two graphs in $S^{3}$ that appear in Figure 5 will work.
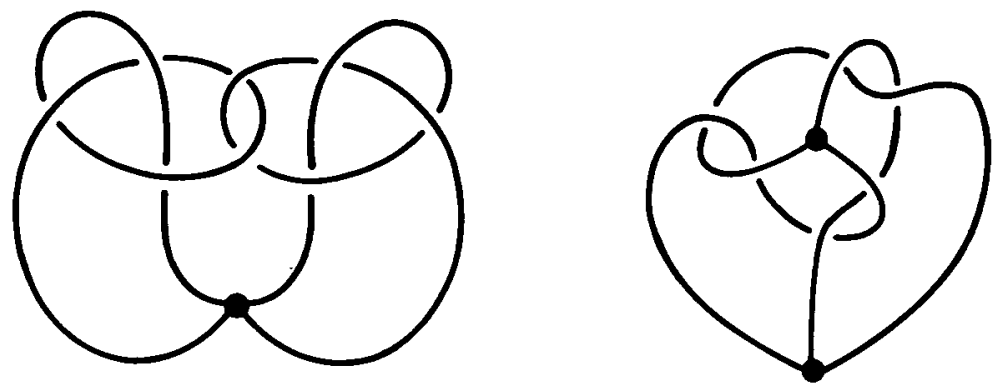

FIGURE 5

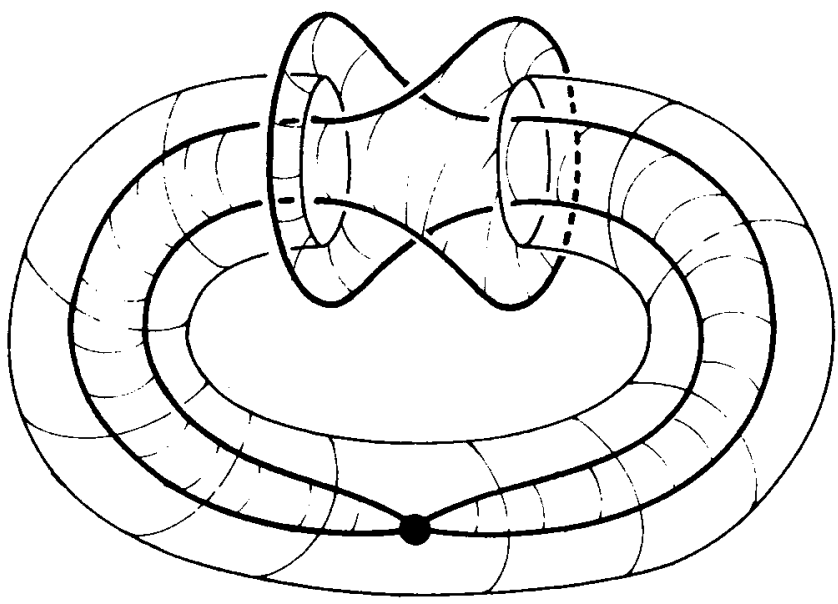

FIGURE 6

Denote these two handlebody complements by $W_{1}$ and $W_{2}$ respectively. The first of these is stated to have the requisite properties in [6, p.194], and proved to have them in [12]. Although the second graph appearing in [6, p.194] supposedly has the appropriate properties, it actually does not. The corresponding 
handlebody complement does contain an essential annulus as in Figure 6, which we discovered with some help from Jeff Week's program SNAPPEA.

The manifold $W_{2}$ is the so-called tripos manifold, described in Chapter 3 of [17]. It can be obtained by gluing together two truncated tetrahedra, each with dihedral angles $\pi / 6$, along pairs of faces. This is one of eight manifolds that Kojima and Miyamoto prove to be the smallest closed hyperbolic manifolds with totally geodesic boundary (cf. [7]).

Two particular choices of how to embed $\left(H, K_{1}\right)$ in $S^{3}$ so that the closure of the complement of $H$ is each one of $W_{1}$ and $W_{2}$ yield the two knots in Figure 7.

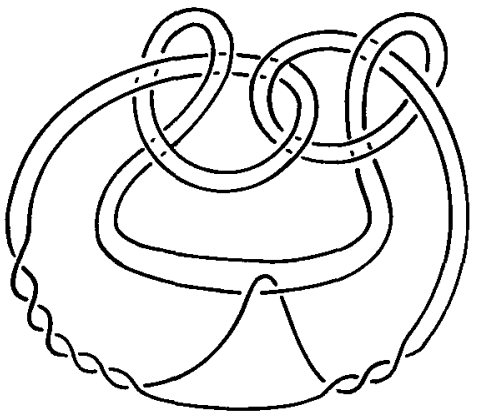

(a)

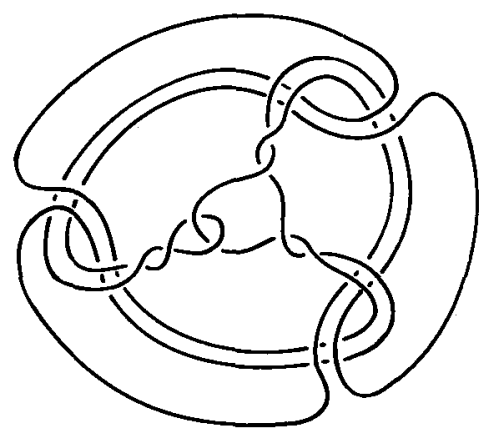

(b)

FIGURE 7. (a) Vol $=22.42567$ (b) Vol $=18.02997$

The computer program SNAPPEA was utilized to attempt to find the particular embeddings so that the resultant knot complements had the least volume possible. Since $W_{1}$ and $W_{2}$ are both acylindrical, the two resulting knot complements are both hyperbolic and each contains an incompressible genus two surface that carries no accidental parabolics. Since $H-K_{1}$ is not acylindrical, the incompressible surface cannot be totally geodesic in the knot complement. Hence, each of these knots has a non-Fuchsian but quasi-Fuchsian surface in its complement. Any of the other knots that would result from some other choice of how to embed $\left(H, K_{1}\right)$ in $S^{3}$ such that the closure of its complement is $W_{1}$ or $W_{2}$ would also have a hyperbolic complement containing an incompressible non-Fuchsian quasi-Fuchsian surface.

In Figure 8 we display two knots resulting from two choices of how to embed $\left(H, K_{2}\right)$ in $S^{3}$ so that the complement of the interior of $H$ is one of the two manifolds $W_{1}$ and $W_{2}$. Again, we have utilized SNAPPEA to try to find the embeddings that minimize the volume of the resulting knot complement. 


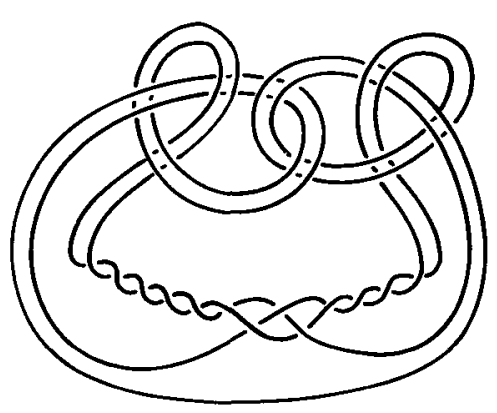

(a)

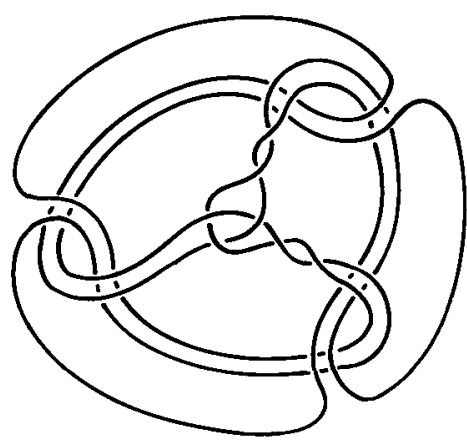

(b)

FIGURE 8. (a) Vol $=24.59305$ (b) $\mathrm{Vol}=21.21917$

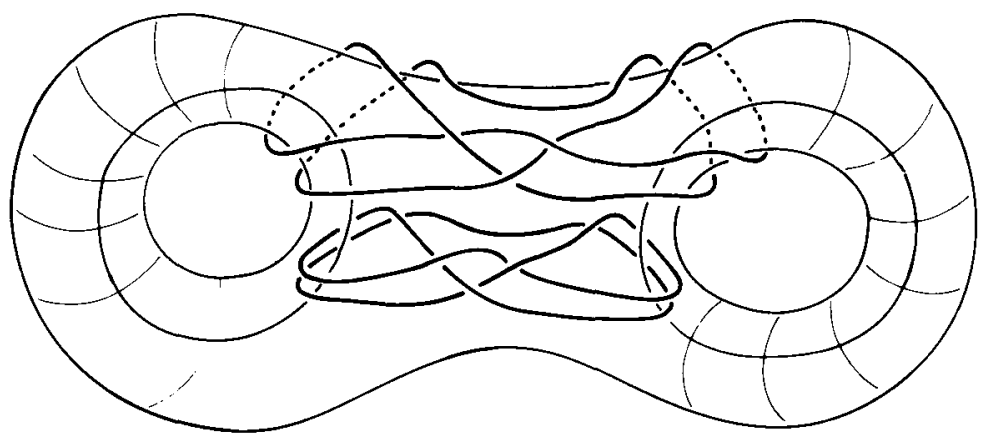

FIGURE 9

Here, since both $H-K_{2}$ and $W_{i}$ are acylindrical, the incompressible genus two surface could appear as a totally geodesic surface in the hyperbolic structure on $S^{3}-K$. However, by doubling $\left(H, K_{2}\right)$ along the boundary of $H$, we obtain a two component link in $\left(S^{2} \times S^{1}\right) \#\left(S^{2} \times S^{1}\right)$. Representing this as a Dehn surgery with slope 0 along two components of a four component link in $S^{3}$, as in Figure 9, we can utilize SNAPPEA in order to find the volume of the double of $H-K_{2}$. (The doubling surface is also depicted in Figure 9.)

Half of this then gives the volume of $\mathrm{H}-\mathrm{K}_{2}$ when the boundary of $\mathrm{H}$ is totally geodesic. The resulting volume is $12.046092040094 \ldots$. . Since Kojima and Miyamoto proved that a compact hyperbolic manifold with totally goedesic boundary must have volume at least $6.451990270835 \ldots$, we see that 


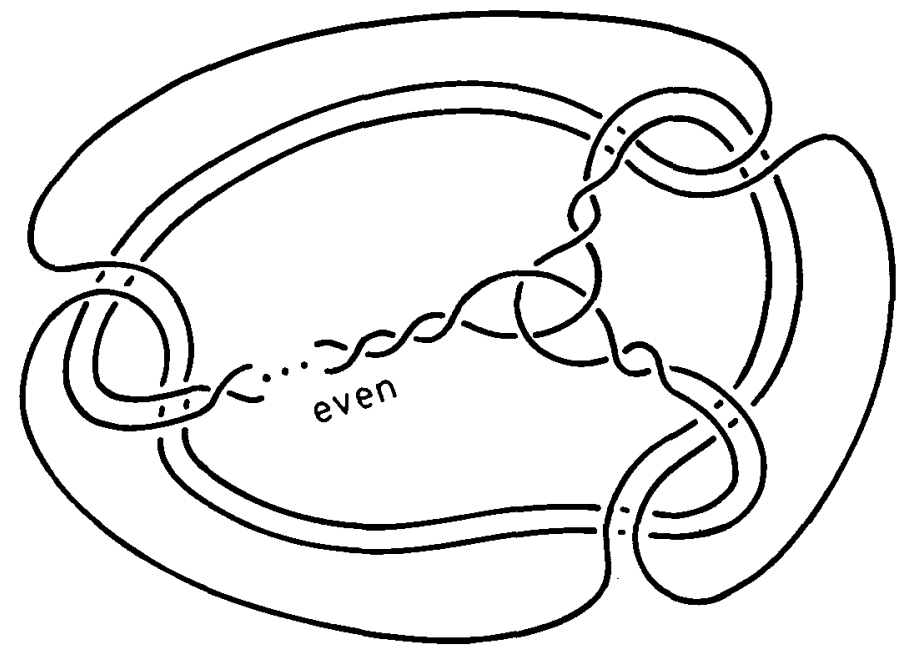

FIGURE 10

if the incompressible surface were to remain totally geodesic after $\mathrm{H}-\mathrm{K}_{2}$ is glued to $W_{i}$, the volume of the resulting manifold would have to be at least $18.49808267177 \ldots$. In fact, the tripos manifold realizes the lower bound on volume of Kojima and Miyamoto and therefore when $i=2$, the volume of the resulting manifold would have to be exactly $18.49808267177 \ldots$. Since this is not the volume of the second knot complement, the essential surface is not totally geodesic in this knot complement.

When $W_{1}$ is given a hyperbolic metric so that the boundary is totally geodesic, similar techniques can be applied in order to use SNAPPEA to find the volume. The result is $8.093993534733 \ldots$... Since the volume of the corresponding knot complement is greater than the sum of this volume and the volume of $\mathrm{H}-\mathrm{K}_{2}$, the incompressible surface again cannot be totally geodesic in the knot complement.

The sequence of knots in Figure 10 all come from $(1, p)$-Dehn surgery on the new component of a link obtained by encircling the two strands that are being twisted about one another by a trivial component. By our work above, all of these knots are hyperbolic. Since the link that we are performing surgery on is hyperbolic, there exists an $N$ such that for all $i \geq N$, the sequence of volumes of the knot complements approaches the volume of the link from below monotonically (cf. [14, 16]).

In particular, this means that the set of $i \geq N$ yields an infinite set of knot complements, all of which have distinct volumes. Since each can be cut open 
along the incompressible surface to yield the same two submanifolds, and since the hyperbolic volumes of those two submanifolds corresponding to when the boundary surface is totally geodesic are completely determined, it must be the case that at most one of these knot complements can contain this surface as a totally geodesic surface.

Explicit calculations with Jeff Week's SNAPPEA program empirically point to the conclusion that the least volume corresponds to the knot depicted in Figure 7(b). A proof of this fact would imply that not even one of these knot complements could contain the incompressible genus two surface as a totally geodesic surface, as the least volume of any of these knot complements would be greater than $18.498 \ldots$.

In closing, we note that a result of Wu implies at most two non-trivial surgeries on either of the knots depicted in Figure 8 will yield a manifold in which th essential surface compresses (cf. [19, Theorem 1]). (In fact, the offending surgeries must be $(p, 1)$ and $(p+1,1)$-surgeries.) For $i=1,2$, an examination of the shape of the cusp by SNAPPEA shows that the only homotopy class of a simple closed curve in the boundary of a maximal cusp with a representative of length less than $2 \pi$ is a meridian. The $2 \pi$ Theorem of Gromov and Thurston (cf. $[5,3]$ for a description and proof) then implies that any non-trivial surgery on one of these knots yields a negatively curved manifold. However, a negatively curved manifold that contains an essential surface must be hyperbolic. Thus, all but at most two non-trivial surgeries on each of these knot complements yield closed Haken hyperbolic 3-manifolds.

\section{References}

[1] C. Adams, 'Toroidally alternating knots and links', preprint, 1992.

[2] C. Adams, J. Brock, J. Bugbee, T. Comar, K. Faigin, A. Huston, A. Joseph and D. Pesikoff, 'Almost alternating links', Topology Appl. 46 (1992), 151-165.

[3] S. Bleiler and C. Hodgson, 'Spherical space forms and Dehn filling', preprint, 1991.

[4] J. H. Conway, 'An enumeration of knots and links and some of their algebraic properties', in: Computational Problems in Abstract Algebra (Pergamon Press, New York, 1970) pp. 329-358.

[5] M. Gromov and W. Thurston, 'Pinching constants for hyperbolic 3-manifolds', Invent. Math. 89 (1987), 1-12.

[6] W. Jaco, Lectures on three-manifold topology, Regional Conference Series in Mathematics 43 (Amer. Math. Soc., Providence, 1977).

[7] S. Kojima and Y. Miyamoto, 'The smallest hyperbolic 3-manifolds with totally geodesic boundary', J. Differential Geom. 34 (1991), 175-192. 
[8] M. T. Lozano, 'Arcbodies', Math. Proc. Cambridge Philos. Soc. 94 (1983), 252-260.

[9] M. T. Lozano and J. H. Przytycki, 'Incompressible surfaces in the exterior of a closed 3-braid I, surfaces with horizontal boundary components', Math. Proc. Cambridge Philos. Soc. 98 (1985), 275-299.

[10] B. Maskit, Kleinian Groups (Springer-Verlag, Berlin, 1987).

[11] W. Menasco and A. Reid, 'Totally geodesic surfaces in hyperbolic link complements', in: Topology '90 (eds. B. Apanasov, W. Neuman, A. Reid and L. Siebenmann) (de Gruyter, Amsterdam, 1992).

[12] R. Myers, 'Simple knots in compact, orientable 3-manifolds', Trans. Amer. Math. Soc. 273 (1982), 75-91.

[13] W. Neumann and A. Reid, 'Rigidity of cusps in deformations of hyperbolic 3-orbifolds', preprint.

[14] W. Neumann and D. Zagier, 'Volumes of hyperbolic 3-manifolds', Topology 24 (1985), 307-332.

[15] U. Oertel, 'Closed incompressible surfaces in complements of star links', Pacific J. Math. 111 (1984), 209-230.

[16] W. P. Thurston, The geometry and topology of 3-manifolds (Lecture notes, Princeton University, 1978).

[17] - The geometry and topology of 3-manifolds (Lecture notes, Berkeley edition, 1992).

[18] J. Weeks, 'SNAPPEA, the hyperbolic structures computer program', see note (1992). (For a description, see C. Adams, SNAPPEA: The Week's hyperbolic 3-manifolds program, Notices Amer. Math. Soc. 37 (1990), 273-275).

[19] Y-Q. Wu, 'Incompressibility of surfaces in surgered 3-manifolds', Topology 31 (1992), 271-280.

Department of Mathematics

Williams College

Williamstown, MA 01267

USA

e-mail: adams@cc.williams.edu
Mathematical Sciences Research Institute 1000 Centennial Drive Berkeley, CA 94720 USA email: reid@msri.org 